\title{
High pre-transplant FGF-23 level is associated with poor graft survival and persistent vitamin $D$ insufficiency in kidney transplant patients
}

\author{
JungHwa Ryu', Hee Jung Jeon ${ }^{2}$, Tae Yeon Koo ${ }^{3}$, Jaeseok Yang ${ }^{4}$

\footnotetext{
${ }^{1}$ Department of Internal Medicine-Nephrology, Ewha Womans University Seoul Hospital, Seoul, Korea

${ }^{2}$ Department of Internal Medicine-Nephrology, Hallym University College of Medicine, Seoul, Korea

${ }^{3}$ Department of Internal Medicine-Nephrology, Seongnam Citizens Medical Center, Seongnam, Korea

${ }^{4}$ Department of Internal Medicine-Nephrology, Yonsei University College of Medicine, Seoul, Korea
}

Background: Vitamin D [25(OH)D] insufficiency and FGF-23 elevation in chronic kidney disease (CKD) is usually ameliorated after kidney transplantation (KT). However, post-transplant vitamin D insufficiency are still associated with poor graft outcome. This study aimed to investigate the effect of pre-transplant FGF-23 level on post-transplant vitamin D status and clinical outcomes.

Methods: The KoreaN cohort study for Outcome in patients With Kidney Transplantation (KNOW-KT) is a multicenter, observational cohort study. Four hundred subjects for whom serum FGF-23 measurement was available were included in this study. Annual serum 25(OH)D and clinical outcomes; all-cause mortality, cardiovascular event, graft survival, and fracture were assessed according to baseline FGF-23 levels.

Results: Median follow-up was 6.7 years. Serum 25(OH)D levels were increased after KT (before KT, 12.6 $\pm 7.4 ; 1$ year after KT, $22.6 \pm 6.4$; 3 years after $\mathrm{KT}, 24.3 \pm 5.8 \mathrm{ng} / \mathrm{mL}$ ). However, they were declined to $21.2 \pm 8.4 \mathrm{ng} / \mathrm{mL}$ at 6 years after $\mathrm{KT}, 20.6 \pm 8.1 \mathrm{ng} /$ $\mathrm{mL}$. Vitamin D deficiency was present in $79.1 \%$ just before $\mathrm{KT}$, then it was decreased to $30.8 \%$ at 3 years after $\mathrm{KT}$, whereas it was increased $37.8 \%$ at 6 years after KT. Serum FGF-23 level was decreased after KT $(2,140.6 \mathrm{pg} / \mathrm{mL}[391-9,277]$ before KT vs. $50.0 \mathrm{pg} / \mathrm{mL}$ [23.6-94.6] at 3 years after $\mathrm{KT} ; \mathrm{P}=0.001$ ). The FGF-23 showed negative correlation with serum vitamin $\mathrm{D}$ levels. When we categorized subjects into tertile according to baseline GFG-23 level; low, middle, high FGF-23 groups. However, the 25(OH)D in the low baseline FGF-23 group was lowest at any point during follow-up. High baseline FGF-23 level was a risk factor for poor graft survival (hazard ratio, 2.098; 95\% confidence interval, 1.201-3.664; $P=0.009$ ).

Conclusions: Increased FGF-23 could interfere vitamin D activation even after KT and is a risk factor for graft survival.

Corresponding author: Jaeseok Yang

E-mail: jcyjs@snu.ac.kr

(c) The Korean Society for Transplantation

This is an Open Access article distributed under the terms of the Creative Commons Attribution Non-Commercial License (http://creativecommons.org/licenses/by-nc/4.0/) which permits unrestricted non-commercial use, distribution, and reproduction in any medium, provided the original work is properly cited. 